\title{
Evaluating the impact of an instructional resource in pharmaceutical calculations for final year pharmacy students
}

Authors: Cleopatra Branch and Ruth Rodgers. Medway School of Pharmacy, Universities of Kent \& Greenwich

Background: The ability to perform pharmaceutical calculations accurately and competently is an essential skill that every pharmacist must possess to ensure patient safety. It is therefore vital that undergraduate students are taught the necessary calculation skills from tertiary level entry. More importantly, students should retain these skills as they matriculate through the curriculum, to ensure competency in calculations at the point of graduation and in actual practice.

Aim: To pilot and evaluate an instructional resource developed to motivate final year students' learning in pharmaceutical calculations.

Method: The learning resource consisted of formative assessments, a face-to-face tuition session and a novel weekly 'online text' assignment with an interactive discussion forum, via the University learning and teaching platform (Moodle). Students completed a post-module satisfaction questionnaire.

Results: A total of 113 final year students completed questionnaires, yielding a response rate of $96 \%$. The instructional resource, especially the Moodle-based interactive tuition (Moodle Thursday), was well accepted by students, with 93\% strongly agreeing or agreeing that it was helpful, and $61 \%$ felt that it contributed most to their learning. 'Moodle Thursday helped to develop and improve my understanding, logic and confidence in pharmaceutical calculations' Students appreciated the individual feedback on each Moodle Thursday assignment and the fact that they were able to practise calculations in their 'own time' and learn at their 'own pace'. They opted for the weekly Moodle based tuition to be continued but suggested that the number of calculation questions in the weekly problem set be increased.

Conclusion: The instructional resource, appeared to have increased students' understanding and confidence in performing pharmaceutical calculations, and encouraged self-directed learning. The positive students' feedback subsequently led to its implementation in the final year curriculum. 